\title{
Saphenous Nerve
}

National Cancer Institute

\section{Source}

National Cancer Institute. Saphenous Nerve. NCI Thesaurus. Code C147511.

A branch of the femoral nerve traveling the length of the leg/hindlimb, which innervates the sartorius and the skin of the medial aspect of the leg/hindlimb from the knee/stifle joint to the metatarsus. 